\title{
Photomechanical energy conversion based on cross-linked liquid-crystalline polymers
}

\author{
Jun-ichi Mamiya \\ An actuator is an energy transducer that can convert input energies into work. Cross-linked liquid-crystalline polymers show \\ photoinduced deformation with changes in the molecular shape and alignment of the polymers. Polymer materials can \\ transduce converted light energy into mechanical stress by the macroscopic deformation of the polymers (photomechanical \\ effect). The effect of the molecular structures of the photochromic liquid-crystalline polymers on the photoinduced deformation \\ is studied. The mechanism of the photoinduced deformation of the polymers is investigated. Three-dimensional movements of \\ the liquid-crystalline polymers are achieved by laminating the polymers into a flexible polymer sheet. In this review, the design \\ of the polymer materials with a photochromic moiety and an evaluation of their photomechanical effects are described. The \\ photomodulation of the alignment of liquid-crystalline polymers and their applications for light-driven polymer actuators are \\ summarized.
}

Polymer Journal (2013) 45, 239-246; doi:10.1038/pj.2012.140; published online 1 August 2012

Keywords: actuator; cross-linked polymer; energy conversion; liquid crystal; photochromism; photomobile material

\section{INTRODUCTION}

Light is a clean energy that can be rapidly, precisely and remotely controlled. The efficient use of light energy is necessary for establishing a sustainable society. Light-responsive materials have been of great interest from the viewpoint of optical memory, ${ }^{1-4}$ solar cells ${ }^{5}$ and light-driven actuators. ${ }^{6}$ An actuator is an energy transducer that can convert a variety of input energies to mechanical quantities, such as displacement, strain, velocity and stress. ${ }^{7-11}$ To duplicate human muscle-like movements in artificial actuators, they should be soft and deform in response to external stimuli, such as electric fields, temperature and light. The design and development of various types of molecular machines have been extensively studied, and machine-like motions have been well characterized using spectroscopic analyses. ${ }^{12-15}$ However, this effect was too small to be utilized in practical applications. Macroscopic mechanical work is desired in actuators or molecular machines. Recently, photoinduced macroscopic motions of organic crystals and amorphous materials consisting of photochromic compounds have been demonstrated. ${ }^{16-24}$ The photoinduced movement of a two-component cocrystal composed of diarylethene derivatives has been reported. ${ }^{16}$ The millimeter-size crystals exhibited macroscopic mechanical work in the form of light-driven actuators.

Polymer materials meet the requirements of artificial muscles because of their advantageous properties: high processability, flexibility, easy fabrication characteristics and low manufacturing costs. Many materials are used as polymer actuators. ${ }^{6-8,11,25-27}$ Shapememory polymers are materials that can be deformed and fixed into a temporary shape; only after being exposed to an appropriate stimulus will they recover their original shape. ${ }^{28,29}$ The photoresponsive property can be introduced into shape-memory polymers by incorporating various functional groups. Lendlein et al. ${ }^{29}$ reported the synthesis of photoinduced shape-memory polymers containing cinnamic acid groups. Exposure of these polymers to UV light stabilized the elongated shape by a photoinduced $[2+2]$ cycloaddition reaction after the polymer film was elongated by an external force. The deformation of polymer colloid particles by exposure to light was also reported by Wang et al. ${ }^{30,31}$ The spherical polymer particles containing azobenzene moieties changed from a sphere into an ellipsoid upon exposure to interfering linearly polarized laser beams. The elongation of the particles was induced along the polarization direction of the laser beam.

Liquid-crystalline elastomers (LCEs) are one of the most promising polymer actuator materials because of their unique property of combining the anisotropy of the LCs with the elasticity of the polymer networks. ${ }^{32}$ The alignment of the mesogens coupled with the polymer networks gives rise to the characteristic properties of LCEs. LCEs are usually lightly cross-linked networks, and the macroscopic properties and phase structures of LCEs are affected by the cross-linking density. LCEs exhibit thermoelastic properties with a nematic-isotropic phase transition; they contract along the alignment direction of the mesogens, and upon cooling below the phase transition temperature, they expand. Kupfer and Finkelmann ${ }^{33}$ reported an anisotropic deformation resulting from the thermal 
LC-isotropic phase transition in monodomain LCEs. The origin of the deformation is a subtle change in the microscopic order after the nematic-isotropic phase transition, and this deformation is closely related to a coupling between the LC order of the mesogens and the elastic properties of the polymer network. This anisotropic deformation behavior of LCEs can be very large and has been a subject of extensive experimental and theoretical studies. ${ }^{34-39}$ Along with their good mechanical properties, LCEs can be promising materials for the development of artificial muscles. ${ }^{40}$

A promising application of the photomechanical effect is the ability to use light to control the expansion and contraction for use as a photoactuator. This photomechanical deformation can be used to create a novel class of photofunctional materials. In this review, we focused on the photoresponsive behavior of the liquid-crystalline polymers. The photocontrol of molecular alignment in LC systems with a photochromic moiety is described. Macroscopic deformation based on a photoinduced change in the molecular structure and the alignment of the cross-linked LC polymers is introduced. Recent intensive studies of light-driven actuators using cross-linked LC polymers are summarized.

\section{PHOTOINDUCED CHANGE IN THE MOLECULAR ALIGNMENT OF LIQUID-CRYSTALLINE POLYMERS}

Photochromism is defined as a reversible photoinduced transformation of a molecule between two isomers whose absorption spectra are distinguishably different. ${ }^{41}$ The physical properties of photochromic molecules can be controlled using light. Azobenzene, which is a common photochromic molecule, exhibits reversible trans-cis isomerization induced by light. ${ }^{4,42,43}$ The trans form of azobenzene has a rod-like shape, which stabilizes the phase structure of the LC phase, whereas the cis form, which has a bent shape, destabilizes the phase structure of the mixture. A small amount of photochromic molecules are incorporated into the LC molecules, and the mixtures are irradiated. ${ }^{44-46}$ The photoinduced change in the molecular shape of the azobenzenes causes a change in the alignment of the surrounding LC molecules, and an LC-isotropic phase transition is induced. During cis-trans back isomerization of the azobenzene, the mixture returns to the initial LC phase. This process means that the change in the molecular alignment is amplified by the cooperative motion (Figure 1a). LC polymers possess properties of both polymers and LCs, and they are currently regarded as promising photonic materials because of their advantageous properties. In azobenzene derivatives exhibiting LC properties, the azobenzene moiety could act as both a mesogen and a photoresponsive moiety. Azobenzene LC polymers undergo a nematic-isotropic phase transition upon photoirradiation. The irradiated site becomes isotropic, as evidenced by the loss of birefringence when observed with a polarizing microscope. The stored image has been stabilized below the glass transition temperature (Tg) of the polymer. If the temperature is raised above the Tg, the light-induced molecular alignment is erased and returned to its initial state because of the molecular motion of the main chain in the polymer. The alignment of the mesogens is unlikely in the absence of segmental motions of the polymer main chain below the Tg. Therefore, the azobenzene LC polymers can be utilized as optical switching and optical image-storage materials. ${ }^{47}$

Trans azobenzenes that have transition moments parallel to the polarization direction of linearly polarized light are effectively activated to their excited states, which is followed by trans-cis isomerization, whereas molecules that have their transition moments perpendicular to the polarization direction of actinic light are inactive. After repeating the trans-cis-trans isomerization cycles and a
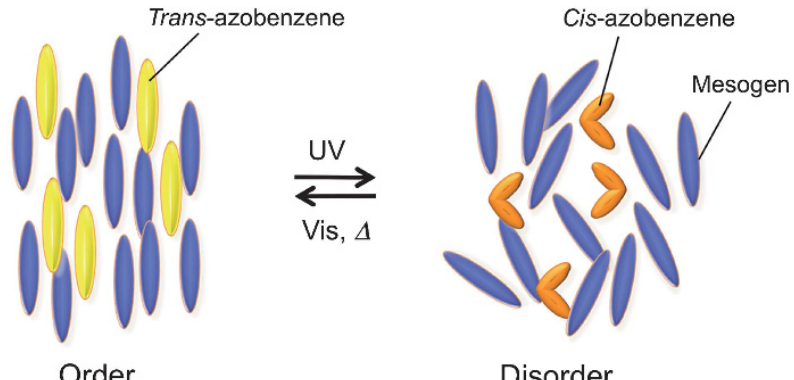

Disorder

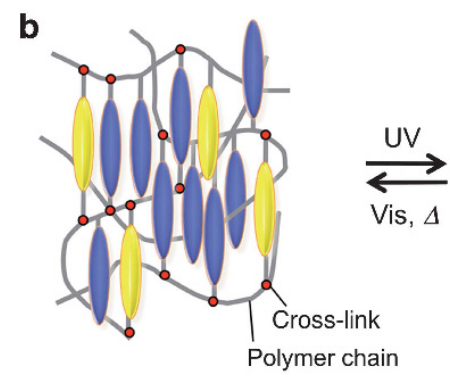

Order

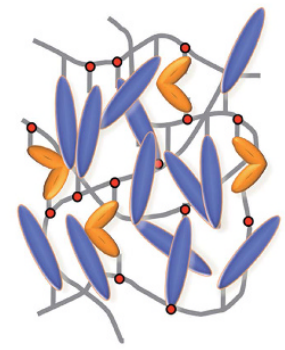

Disorder

Figure 1 Photochemical phase transition of LCs doped with azobenzenes (a) and cross-linked LC polymers (b).

after the trans-azobenzene molecules have adapted an orientation perpendicular to the polarization direction of the actinic light, they become inactive towards the incident light. This result means that at the end of multicycles, there will be a net population of azobenzene molecules aligned perpendicular to the polarization direction of the light. This light-induced effect results in anisotropy in the molecular alignment of dye molecules. ${ }^{48,49}$

Azobenzene molecules have been almost exclusively employed in photochromism-based holographic gratings, mainly because the photoisomerization of azobenzenes enables alignment changes of the LC molecules quite effectively, as mentioned above. ${ }^{4,50}$ If the alignment change in a periodic structure is induced in accordance with the interference patterns produced by overlapping two coherence beams, a large refractive-index modulation in LC materials can be obtained. ${ }^{51-53}$ The diffraction efficiency is strongly related to the refractive-index modulation produced in the materials. In photochromic polymer films, surface-relief structures are formed on the surfaces of the polymers and refractive-index modulation occurs upon irradiation with two coherent laser beams (Figure 2). The sinusoidal variation in light intensity is encoded into the materials as a sinusoidal surface topology, that is, a surface-relief grating (SRG). ${ }^{42,54,55}$ The original film thickness and flat topology can be recovered by heating the polymer films above their Tg. During this patterning process, the polymer materials are moved over nanometerto micron-length scales at temperatures below their $\mathrm{Tg}$. This phenomenon can be primarily regarded as a manifestation of the photomechanical effect. ${ }^{56}$

Cross-linking of the polymer chains in LC polymers results in a strong correlation between the functional groups that are attached to the LC polymers, which results in an anomalous responsive behavior to external stimuli. Kurihara et al. ${ }^{57}$ prepared cross-linked LC polymers with an azobenzene moiety and evaluated their photoresponsive behavior. The response time is fast $(1 \mu \mathrm{s})$ because the motion of the mesogenic groups is suppressed by cross-linking. The stabilization of an initially ordered state by cross-linking results in 


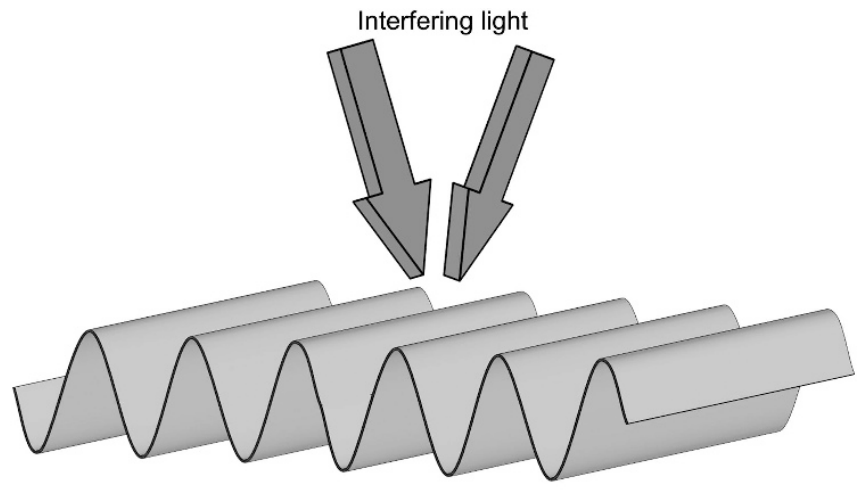

Surface relief structures on the polymer surface

Figure 2 Schematic illustration of the surface-relief grating of LC polymers upon irradiation with two coherent laser beams.

a fast order-disorder transition, which is induced by slight changes in the orientational order of the mesogens, and a fast disorder-order transition, which is caused by the strain relaxation that occurs during the photoisomerization of the azobenzene molecules (Figure 1b). ${ }^{58,59}$ Polymer gels with azobenzenes deform because of photoinduced structural changes in the azobenzenes. Photochromic materials with network structures show changes in various properties during the isomerization of the compounds. The molecular-level photoisomerization can also result in macroscopic deformation of the material system, allowing one to convert light energy directly into mechanical work (photomechanical materials).

\section{PHOTOMECHANICAL EFFECTS OF LIQUID-CRYSTALLINE POLYMERS}

As described in the introduction, LCEs exhibit thermoelastic properties; when heated above the nematic-isotropic phase transition, LCEs contract along the alignment direction of the mesogens, and by cooling below the phase transition temperature, they expand. By combining the thermoelastic properties of LCEs with a photochemical phase transition (or a photochemically induced reduction of nematic order), it is expected that one can induce the deformation of LCEs with light. ${ }^{60-62}$ In fact, $20 \%$ contraction of an azobenzene containing an LCE that has a polysiloxane main chain was induced upon exposure to UV light, which caused a trans-cis isomerization of the azobenzene moiety, as demonstrated by Finkelmann. Subtle changes in the nematic order during trans-cis isomerization results in a significant uniaxial deformation of the LCs along the director axis when the LC molecules are strongly associated by covalent crosslinking to form a three-dimensional polymer network.

Ikeda et al. ${ }^{63}$ observed photoinduced three-dimensional movements of LC gels and cross-linked LC polymers ${ }^{64}$ that contained azobenzenes. In comparison with the contraction mode, which is a two-dimensional movement, the bending mode, which is a threedimensional movement, could be advantageous for artificial hands and microrobots that are capable of particular manipulations. Figure 3a shows the bending and unbending processes induced by irradiation with UV and visible light, respectively. It was observed that the monodomain LC polymer film bent toward the irradiation direction of the incident UV light along the rubbing direction, and the bent film reverted to its initial flat state after exposure to visible light. This bending and unbending behavior was reversible simply by changing the wavelength of the incident light. The bending direction was also controlled by the alignment direction of the mesogens (Figure 3b). ${ }^{65,66}$

Freestanding cross-linked LC polymer films were prepared by selfassembly through intermolecular hydrogen bonding. The LC polymer films with hydrogen-bonded network structures exhibited photoinduced bending. The structural change at the microscopic level, which is caused by the trans-cis photoisomerization of the azobenzene moieties at the hydrogen-bonded cross-links, is successfully converted to a macroscopic deformation in the films. A supramolecular photomechanical system based on hydrogen-bonded LC polymer films, which converts light energy directly into mechanical work, was achieved. $^{67}$

Photomobile polymer materials based on the cross-linked LC polymers deformed by disordering the molecular alignment through the photoisomerization of the photochromic moiety and motion of the polymer chain. Mechanical stress generated by the deformation of the polymers was evaluated. The cross-linked LC polymers were prepared by the photopolymerization of mixtures with different ratios of the monomer and the cross-linker. The mechanical stress increased with the increase in the cross-linking concentration. This result indicates that an increase in the cross-linking point caused an amplification of the change in molecular alignment, which results in macroscopic deformation of the cross-linked LC polymers. Not all mesogens are photochromic molecules. By improving the penetration of incident light into the polymer films, higher performance of the photomobile materials would be expected. As the feed ratio of azobenzene moieties into the polymer films decreases, the degree of the isomerization and the penetration depth of incident light are enhanced, which leads to an effective photoinduced movement. The mechanical stress generated in the polymers was 10 times higher than that of natural muscles. ${ }^{68}$ Further, no degradation of the polymers occurred, even when the photoinduced bending was repeated over 5000 times. ${ }^{69}$

Irradiation with UV light results in the trans-cis isomerization of the azobenzene moieties and destabilization of the LC phase (reduction in LC order), even the LC-isotropic phase transition in the LC systems. Because the extinction coefficient of the azobenzene moieties at $\sim 360 \mathrm{~nm}$ is large, the reduction in LC order occurs only in the surface region that faces the incident light, but in the bulk of the film, the trans-azobenzene moieties remain unchanged. As a result, the volume contraction is generated only in the surface layer, which causes the bending to occur towards the irradiation direction of the incident light (Figure 4). The process is highly sensitive to the initial alignment of the mesogens. However, the detailed relationship between the molecular alignment of the mesogens and photoinduced bending is unclear. Changes in birefringence and mechanical stress in the polymer films were investigated simultaneously to evaluate the relationship between the molecular alignment of the mesogens and the contraction of the films upon photoinduced bending. An azobenzene cross-linker, a nonphotoactive monomer and a nonphotoactive cross-linker were used to prepare polymer films with different cross-linker concentrations and a 5-mol\% azobenzene concentration (Figure 5). All of the polymer films with different cross-linker concentrations bent toward the light source upon irradiation with UV light and reverted to their initial state upon irradiation with visible light. When the cross-linker concentration was increased, the degree of bending in the films decreased. These results indicate that the flexibility of the polymer films decreases because of the increase in the cross-linker concentration. The experimental setup for evaluating the photoinduced changes in birefringence and stress is shown in Figure 6. The intensity of a probe beam from a He-Ne laser was 

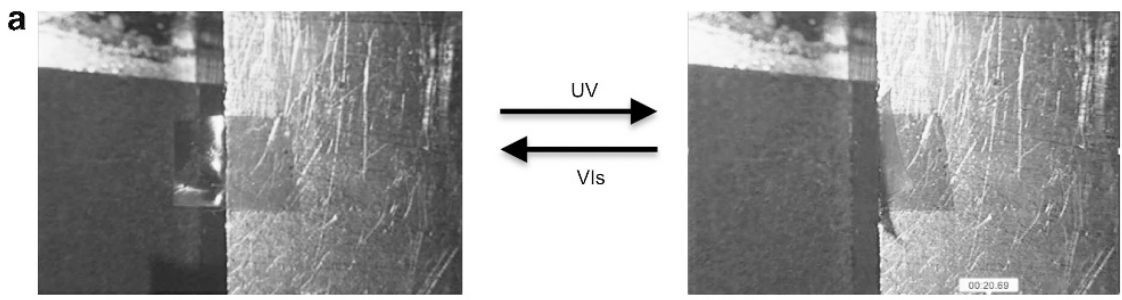

b
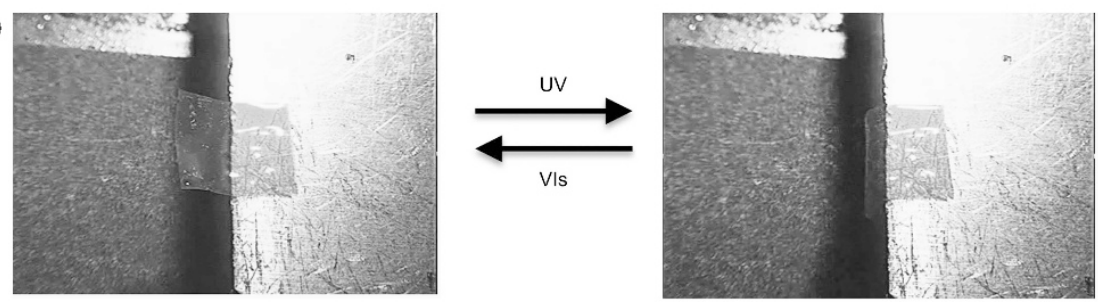

Figure 3 Photographs of the photoinduced bending of the cross-linked LC polymer films with homogeneous (a) and homeotropic (b) alignment of the mesogens.
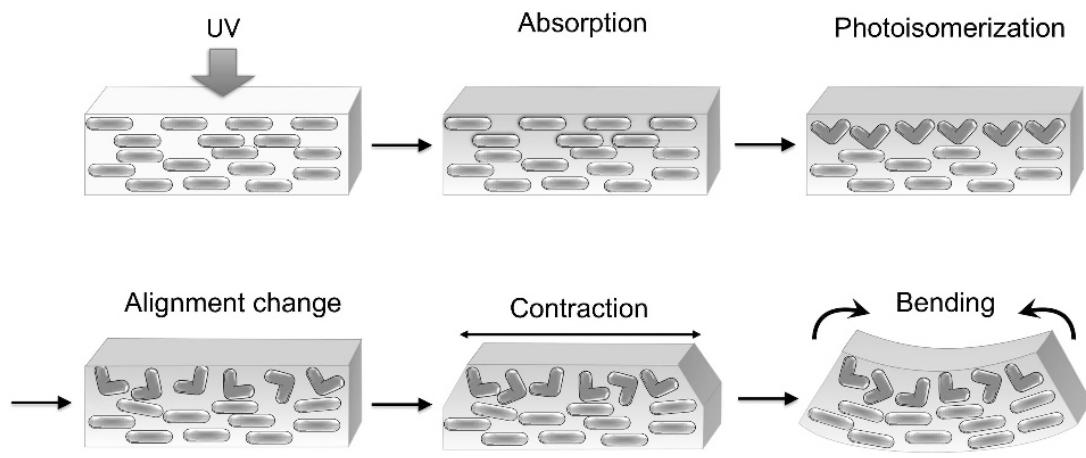

Figure 4 Plausible mechanism of anisotropic bending in the cross-linked LC polymer films upon irradiation with UV light.

a Azobenzene derivatives<smiles>C=CC(=O)OCCCCC(C)(C)Oc1ccc(N=Nc2ccc(OCC(C)(C)C)cc2)cc1</smiles>

K 67 Sm 94 I (Heating)

K 60 Sm 92 I (Cooling) b<smiles>C=CC(=O)OCC(C)(C)COc1ccc(N=Nc2ccc(OC(C)(C)C(C)(C)C(=O)OC(=O)C=C)cc2)cc1</smiles>

K 84 Sm 93 I (Heating)

K 74 Sm 92 I (Cooling)

\section{Phenyl benzoate derivatives}<smiles>C=CC(=O)OCCCC(C)(C)Oc1ccc(C(=O)Oc2ccc(OCC(C)C)cc2)cc1</smiles>

K 79 I (Heating)

K 45 Sm 70 I (Cooling) d<smiles>C=CC(=O)OCC(C)(C)COc1ccc(C(=O)Oc2ccc(OC(=O)c3ccc(OC(C)(C)C(C)(C)C(C)(C)C)cc3)c(C)c2)cc1</smiles>

K 63 N 108 I (Heating)

- N 108 I (Cooling)

Figure 5 Chemical structures of the azobenzene monomer, azobenzene cross-linker, phenyl benzoate monomer and phenyl benzoate cross-linker.

monitored using a photodiode through a sample between crossed polarizers. The photoinduced stress was measured using a thermomechanical analyzer by clamping the films at both ends and using an initial load of $10 \mathrm{mN}$ in the mesogen alignment direction. The photoinduced change in the birefringence was estimated from the transmittance of the probe light. Figure 6 shows the simultaneous measurement of photoinduced changes in the birefringence and photoinduced stress. ${ }^{70}$ The generated stress and the birefringence changes followed the same time dynamics. This result indicates that the disorder of the molecular alignment induced by the trans-cis photoisomerization of the azobenzene moieties causes a mechanical stress in the cross-linked LC polymers. The photoinduced stress exhibited a clear dependence on the cross-linker concentration. This result indicates that stiffer polymer materials with moderate 

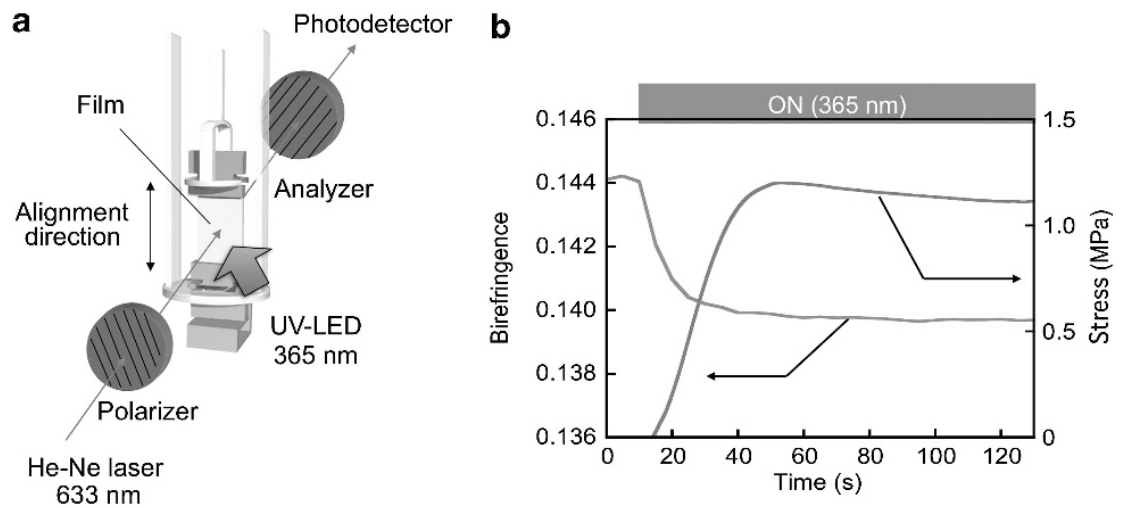

Figure 6 (a) Schematic illustration of the experimental setup for evaluating the photoinduced change in the molecular alignment and photoinduced stress of the samples. (b) Photoinduced stress and change in birefringence of the samples upon UV irradiation.

azobenzene concentrations are favorable for generating high levels of photoinduced stress.

\section{LIGHT-DRIVEN POLYMER ACTUATORS BASED ON PHOTOMECHANICAL EFFECTS}

If the cross-linked LC polymers with photomechanical properties are applied to light-driven actuators, there are many problems, such as mechanical strength, shape and size of the polymers. In a previous study, the photomechanical properties of cross-linked LC polymer films were investigated. By laminating the cross-linked LC polymer films to flexible polymer substrates, large and sophisticated motions of the laminated films can be induced upon photoirradiation. The laminated films were prepared by thermal compression bonding of the cross-linked LC polymer with azobenzene moiety and an unstretched low-density polyethylene film with an adhesion layer, as shown in Figure 7a. By preparing a laminated structure composed a photoactive layer and a flexible polymer substrate, both photoresponsive and good mechanical properties can be provided simultaneously, resulting in complicated movements of the films. ${ }^{71,72} \mathrm{~A}$ plastic belt of the laminated film was prepared by connecting both ends of the film. Simultaneous irradiation of the belt with UV light from the top right and visible light from the top left induced a rotation of the belt to drive two pulleys in a counterclockwise direction at room temperature (Figure 7b). Simultaneously, produced contraction and expansion forces at the different points along the long axis of the belt result in the rotation of the pulleys and the belt with the same direction. ${ }^{71}$ The rotation causes new parts of the belt to be exposed to UV and visible light, which enables the motor system to rotate continuously. New photomechanical devices, including the light-driven plastic motor, were successfully developed using the laminated films. By partially laminating the photoactive layer into a plastic substrate, the laminated films moved freely (similar to a flexible robotic arm) upon photoirradiation (Figure $7 \mathrm{c}$ ). ${ }^{72}$ Lightdriven plastic microrobots consisting of a photoactive polymer layer and a polyethylene film were fabricated. The photoinduced movements of the microrobots were achieved by combining the bilayer film with different initial shapes and photodeformation modes and manipulated to pick up, lift, move and place a milligram-scale object by irradiating different parts of the microrobot with visible light. $^{73}$

All of the polymer microdevices can be fabricated using lithographic techniques in combination with LC polymer actuators. Monomeric LCs with different absorption bands were deposited with an ink-jet printer using separate cartridges on a glass coated with polyvinyl alcohol. The reactive monomers can be cured after printing. The cilia were released from the substrate by dissolving the polyvinyl alcohol layer in water. The microactuators with different subunits can be selectively addressed by changing the wavelength of light. The artificial cilia have the potential to create flow and mixing in wet environments, such as lab-on-a-chip applications. ${ }^{74}$

Cross-linked LC polymer films were prepared from the photopolymerization of aligned LC monomers in a glass cell. This method is useful for preparing films with varying thicknesses and alignments. However, the glass cells must be carefully opened to accommodate freestanding LC films after polymerization. Cross-linking of polymers by means of electron beams (EBs) has been widely used for industrial applications as an alternative to chemical methods for cross-linking in polymers. EB irradiation induced the formation of intra- and interlayer cross-links in the photoactive and substrate layers. Photomobile materials with adhesive-free bilayer structures were fabricated by direct EB irradiation. ${ }^{75}$ After EB irradiation, the polymer films with bilayer structures became almost insoluble in chloroform, indicating the formation of intra- and inter-layer cross-links. The EB-crosslinked polymer films showed a reversible bending, with no fatigue upon photoirradiation. A simple method for fabricating photomobile polymer materials was proposed that can produce large areas, smooth surfaces and controlled thicknesses of both the photoactive and substrate layers.

Human skeletal muscles are composed of many bundles of fibers, and their crucial function to convert energy into mechanical work is achieved by generating smooth motion and inducing high stress with external stimuli. An LC copolymer was mixed with a low-molecularweight cross-linker that contained two isocyanate moieties at the end of molecules, and the mixtures were formed into fibers by dipping a tip of a toothpick into the mixtures and pulling the mixtures with the toothpick as quickly as possible. The polymer fibers showed a high ordering of the mesogens along the fiber axis. When the fibers were heated above the $\mathrm{Tg}$ of the polymers, the fibers contracted along the fiber axis. In addition, the fibers exhibited reversible bending above $90^{\circ} \mathrm{C}$ upon alternative irradiation with UV and visible light. Because the shape of the fibers was approximately cylindrical, the fibers could be irradiated with light under the same conditions from every angle. The direction of the bending could be controlled by changing the irradiation location of the fibers (Figure 8). The directional control in the photomobility of the fibers may lead to potential applications, such as artificial muscles and polymer actuators. ${ }^{76}$ 


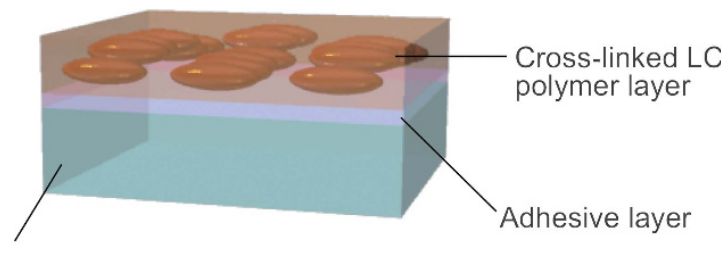

Low-density polyethylene film (PE) $(50 \mu \mathrm{m})$
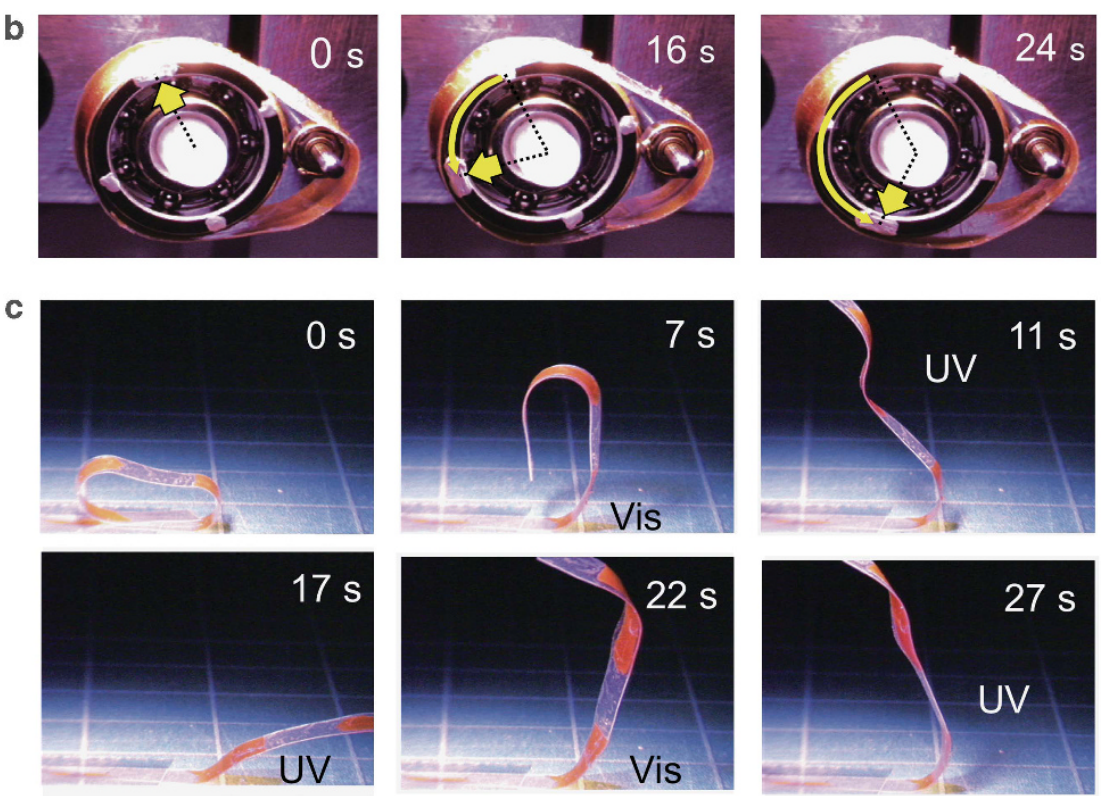

Figure 7 (a) Chemical structures of the LC monomer and cross-linker and a schematic illustration of a laminated film composed of a cross-linked LC polymer layer and a polymer sheet. (b) A light-driven plastic motor with laminated films. Series of photographs showing time profiles of the rotation of the plastic motor with the laminated film induced by simultaneous irradiation with UV and visible light at room temperature. (c) Series of photographs of time profiles of the flexible robotic arm motion.
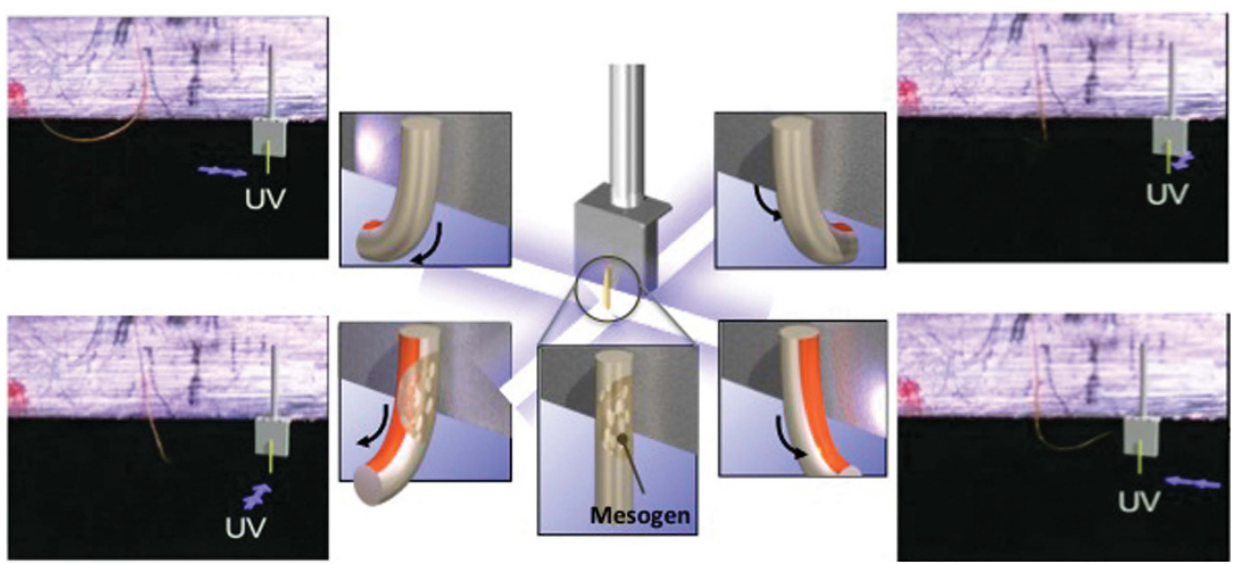

Figure 8 Photographs and schematic illustration of three-dimensional movements of the cross-linked LC polymer fibers by irradiation with UV light.

\section{CONCLUSIONS}

The field of photomechanical effects by means of photochromism is developing rapidly. In this review, recent progress in studies of the photomechanical effect based on cross-linked liquid-crystalline polymers was described. Macroscopic light-driven actuation has been achieved in various systems. Liquid-crystalline polymers that exhibit photochromism can be considered to be promising materials for next-generation applications, such as light-driven and soft actuators. In addition, the actuation performance has been further improved by optimizing the molecular structures and fabrication process with respect to the generated stress and energy-conversion efficiency. Because research activities have increased over the last decade, substantial advances can be expected in the near future.

\section{ACKNOWLEDGEMENTS}

I thank the following colleagues for their guidance and experimental contributions: Professors Tomiki Ikeda, Yanlei Yu, Atsushi Shishido, Drs Motoi Kinoshita, Munenori Yamada, Arri Priimagi, Mizuho Kondo, Aki Shimamura, Yumiko Naka, Mrs Manabu Sugimoto, Akira Yoshitake and Taiki Yoshino. 
1 Yager, K. G. \& Barrett, C. J. in Smart Light-Responsive Materials (eds Zhao, Y. \& Ikeda, T.) Ch. 1 1-46 (Wiley, New Jersey, 2009).

2 Irie, M. Diarylethenes for memories and switches. Chem. Rev. 100, 1685-1716 (2000).

3 Hamp, N. Bacteriorhodopsin as a photochromic retinal protein for optical memories. Chem. Rev 100, 1755-1776 (2000).

4 Ikeda, T. Photomodulation of liquid crystal orientations for photonic applications J. Mater. Chem. 13, 2037-2057 (2003).

5 Gunes, S., Neugebauer, H. \& Sariciftci, N. S. Conjugated polymer-based organic solar cells. Chem. Rev. 107, 1324-1338 (2003).

6 Ikeda, T., Mamiya, J. \& Yu, Y. Photomechanics of liquid-crystalline elastomers and other polymers. Angew. Chem. Int. Ed. 46, 506-528 (2007)

7 Osada, Y., Okuzaki, H. \& Hori, H. A polymer gel with electrically driven motility. Nature 355, 242-244 (1992).

8 Baughman, R. H. Conducting polymer artificial muscles. Synth. Met. 78, 339-353 (1996).

9 Park, S. -E. \& Shrout, T. R. Ultrahigh strain and piezoelectric in relaxor based ferroelectric signal crystals. J. Appl. Phys. 82, 1804-1811 (1997).

10 Baugham, R. H., Cui, C., Zakhidov, A. A., Iqbal, Z., Barisci, J. N., Spinks, G. M., Wallace, G. G., Mazzoldi, A., De Rossi, D., Rinzler, A. G., Jaschinski, O., Roth, S. \& Kertesz, M. Carbon nanotube actuators. Science 284, 1340-1344 (1999).

11 Shahinpoor, M. \& Kim, K. J. Ionic polymer-metal composites: I. Fundamentals. Smart Mater. Struct. 10, 819-833 (2001).

12 Sauvage, J. -P. Transition metal-containing rotaxanes and catenanes in motion: toward molecular machines and motors. Acc. Chem. Res. 31, 611-619 (1998).

13 Balzani, V., Cresi, A., Raymo, F. M. \& Stoddart, J. F. Artificial molecular machines. Angew. Chem. Int. Ed. 39, 3348-3391 (2000).

14 Browne, W. R. \& Feringa, B. L. Making molecular machines work. Nat. Nanotech. 1, 25-35 (2006)

15 Kay, E. R., Leigh, D. A. \& Zerbetto, F. Synthetic molecular motors and mechanical machines. Angew. Chem. Int. Ed. 46, 72-191 (2007).

16 Morimoto, M. \& Irie, M. A diarylethene cocrystal that converts light into mechanical work. J. Am. Chem. Soc. 132, 14172-14178 (2010)

17 Kobatake, S., Takami, S., Muto, T., Ishikawa, T. \& Irie, M. Rapid and reversible shape changes of molecular crystals on photoirradiation. Nature 446, 778-781 (2007).

18 Al-Kaysi, R. O. \& Bardeen, C. J. Reversible photoinduced shape changes of crystalline organic nanorods. Adv. Mater. 19, 1276-1280 (2007).

19 Terao, F., Morimoto, M. \& Irie, M. Light-driven molecular-crystal actuations: rapid and reversible bending of rodlike mixed crystals of diarylethene derivatives. Angew. Chem. Int. Ed. 51, 901-904 (2012)

20 Zhu, L., Al-Kaysi, R. O. \& Bardeen, C. J. Reversible photoinduced twisting of molecular crystal microribbons. J. Am. Chem. Soc. 133, 12569-12575 (2011).

21 Koshima, H., Ojima, N. \& Uchimoto, H. Mechanical motion of azobenzene crystals upon photoirradiation. J. Am. Chem. Soc. 131, 6890-6891 (2009).

22 Koshima, H., Takeuchi, K., Uchimoto, H., Shiro, M. \& Hashizume, D. Photomechanical bending of salicylideneaniline crystals. Chem. Commun. 47, 11423-11425 (2011).

23 Nakano, H. Direction control of photomechanical bending of a photochromic molecular fiber. J. Mater. Chem. 20, 2071-2074 (2010).

24 Nakano, H. \& Suzuki, M. Photoinduced mass flow of photochromic molecular materials. J. Mater. Chem. 22, 3702-3704 (2012).

25 Miyata, T., Asami, N. \& Uragami, T. A reversibly antigen-responsive hydrogel. Nature 399, 766-769 (1999)

26 Pelrine, R., Kornbluh, R., Pei, Q. \& Josoph, J. Hight-speed electrically actuated elastomers with strain greater than 100\%. Science 287, 836-839 (2000).

27 Athanassiou, A., Kalyva, M., Lakioraki, K., Georgiou, S. \& Fotakis, C. All-optical reversible actuation of photochromic-polymer microsystems. Adv. Mater. 17, 988-992 (2005).

28 Lendlein, A. \& Kelch, S. Shape-memory polymers. Angew. Chem. Int. Ed. 41, 2034-2057 (2002)

29 Lendlein, A., Jiang, H., Jünger, O. \& Langer, R. Light-induced shape-memory polymers. Nature 434, 879-882 (2005).

$30 \mathrm{Li}$, Y., He, Y., Tong, X. \& Wang, X. Photoinduced deformation of amphiphilic azo polymer colloidal spheres. J. Am. Chem. Soc. 127, 2402-2403 (2005).

$31 \mathrm{Li}, \mathrm{Y}$., He, Y., Tong, X. \& Wang, X. Stretching effect of linearly polarized $\mathrm{Ar}^{+}$laser single-beam on azo polymer colloidal spheres. Langmuir 22, 2288-2291 (2006).

32 Warner, M. \& Terentjev, E. M. Liquid crystal elastomers (Oxford University Press, Oxford, 2003)

33 Kupfer, J. \& Finkelmann, H. Liquid crystal elastomers: influence of the orientational distribution of the crosslinks on the phase behavior and reorientation processes. Macromol. Chem. Phys. 195, 1353-1367 (1994).

34 Finkelmann, H., Kock, H.-J. \& Rehage, G. Investigation on liquid crystalline polysiloxane 3 liquid crystalline elastomers - a new type of liquid crystalline material. Makromol. Chem. Rapid Commun. 2, 317-322 (1981).

35 Waner, M., Gelling, K. P. \& Vilgis, T. A. Theory of nematic networks. J. Chem. Phys. 88, 4008-04013 (1988)

36 Mao, Y., Terentjev, E. M. \& Warner, M. Cholesteric elastomers: deformation photonic solids. Phys. Rev. E 64, 041803 (2001).

37 Stenull, O. \& Lubensky, T. C. Phase transitions and soft elasticity of smectic elastomers. Phys. Rev. Lett. 94, 018304 (2005).

38 Corbett, D. \& Warner, M. Nonlinear photoresponse of disordered elastomers. Phys. Rev. Lett. 96, 237802 (2006)
39 Crobett, D. \& Warner, M. Linear and nonlinear photoinduced deformations of cantilevers. Phys. Rev. Lett. 99, 174302 (2007).

40 Thomson, III D. L., Keller, P., Naciri, J., Pink, R., Jeon, H., Shenoy, D. \& Ratna, B. R. Liquid crystal elastomers with mechanical properties of a muscle. Macromolecules $\mathbf{3 4}$, 5868-5875 (2001).

41 Irie, M. Photochromism: memories and switches-introduction. Chem. Rev. 100, 1683 (2000).

42 Natansohn, A. \& Rochon, P. Photoinduced motions in azo-containing polymers. Chem. Rev. 102, 4139-4175 (2002).

43 Shibaev, V., Bobrovsky, A. \& Boiko, N. Photoactive liquid crystalline polymer systems with light-controllable structure and optical properties. Prog. Polym. Sci. 28, 729-836 (2003).

44 Sackmann, E. Photochemically induced reversible color changes in cholesteric liquid crystals. J. Am. Chem. Soc. 93, 7088-7090 (1971).

45 Tazuke, S., Kurihara, S. \& Ikeda, T. Amplified image recording in liquid crystal media by means of photochemically triggered phase transition. Chem. Lett. 16, 911-914 (1987).

46 Ikeda, T., Sasaki, T. \& Ichimura, K. Photochemical switching of polarization in ferroelectric liquid-crystal films. Nature 361, 1214-1216 (1993).

47 Ikeda, T. \& Tsutsumi, O. Optical switching and image storage by means of azobenzene liquid-crystal films. Science 268, 1873-1875 (1995).

48 Weigert, F. Uber einen neuen effekt der strahlung in lichtempfindlichen schichten. Verh. Dtsch. Phys. Ges. 21, 479-491 (1919).

49 Todorov, T., Tomova, N. \& Nikolova, L. High-sensitivity material with reversible photoinduced anisotropy. Opt. Commun. 47, 123-126 (1983).

50 Shishido, A. Rewritable holograms based on azobenzene-containing liquid-crystalline polymers. Polym. J. 42, 525-533 (2010).

51 Eich, M., Wendorff, J. H., Rech, B. \& Ringsdorf, H. Reversible digital and holographic optical storage in polymeric liquid-crystals. Makromol. Chem. Rapid Commun. 8, 59-63 (1987)

52 Yamamoto, T., Hasegawa, M., Kanazawa, A Shiono, T \& Ikeda, T. Holographic gratings and holographic image storage via photochemical phase transitions of polymer azobenzene liquid-crystal films. J. Mater. Chem. 10, 337-342 (2000)

53 Yoneyama, Y., Yamamoto, T., Hasegawa, M., Tsutsumi, O., Kanazawa, A., Shiono, T. \& Ikeda, T. Formation of intensity grating in a polymer liquid crystal with a side-chain azobenzene moiety by photoinduced alignment change of mesogens. J. Mater. Chem. 11, 3008-3013 (2001).

54 Natansohn, A. \& Rochon, P. Optically induced surface gratings on azoaromatic polymer-films. Appl. Phys. Lett. 66, 136-138 (1995).

55 Kim, D. Y., Tripathy, S. H., Li, L. \& Kumar, J. Laser-induced holographic surface-relief gratings on nonlinear-optical polymer-films. Appl. Phys. Lett. 66, 1166-1168 (1995).

56 Barrett, C. J., Mamiya, J., Yager, K. G. \& Ikeda, T. Photo-mechanical effects in azobenzene-containing soft materials. Soft Matter 3, 1249-1261 (2007).

57 Kurihara, S., Sakamoto, A. \& Nonaka, T. Fast photochemical switching of a liquidcrystalline polymer network containing azobenzene molecules. Macromolecules $\mathbf{3 1}$, 4648-4650 (1998).

58 Kurihara, S., Sakamoto, A., Yoneyama, D. \& Nonaka, T. Photochemical switching behavior of liquid crystalline polymer networks containing azobenzene molecules. Macromolecules 32, 6493-6498 (1999).

59 Kurihara, S., Yoneyama, D. \& Nonaka, T. Photochemical switching behavior of liquidcrystalline networks: effect of molecular structure of azobenzene molecules. Chem. Mater. 13, 2807-2812 (2001).

60 Finkelmann, H., Nishikawa, E., Pereira, G. G. \& Warner, M. A new opto-mechanical effect in solids. Phys. Rev. Lett. 87, 015501 (2001).

61 Hogan, P. M., Tajbakhsh, A. R. \& Terentjev, E. M. UV manipulation of order and macroscopic shape in nematic elastomers. Phys. Rev. E 65, 041720 (2002).

62 Cviklinski, J., Tajbakhsh, A. R. \& Terentjev, E. M. UV isomerization in nemtic elastomers as a route to photo-mechanical transducer. Eur. Phys. J. E 9, 427-434 (2002).

63 Ikeda, T., Nakano, M., Yu, Y., Tsutsumi, 0. \& Kanazawa, A. Anisotropic bending and unbending behavior of azobenzene liquid-crystalline gels by light exposure. Adv. Mater. 15, 201-205 (2003)

64 Yu, Y., Nakano, M. \& Ikeda, T. Directed bending of a polymer film by light. Nature 425 , 145 (2003)

65 Kondo, M., Yu, M. \& Ikeda, T. How does the initial alignment of mesogens affect the photoinduced bending behavior of liquid-crystalline elastomers? Angew. Chem. Int. Ed. 45, 1378-1382 (2006).

66 Yu, Y., Maeda, T., Mamiya, J. \& Ikeda, T. Photomechanical effects of ferroelectric liquid-crystalline elastomers containing azobenzene chromophores. Angew. Chem. Int. Ed. 46, 881-883 (2007).

67 Mamiya, J., Yoshitake, A., Kondo, M., Yu, Y. \& Ikeda, T. Is chemical crosslinking necessary for the photoinduced bending of polymer films? J. Mater. Chem. 18, 63-65 (2008).

68 Kondo, M., Sugimoto, M., Yamada, M. Naka, Y Mamiya, J., Kinoshita, M., Shishido, A., Yu, Y. \& Ikeda, T. Effect of concentration of photoactive chromophores on photomechanical properties of crosslinked azobenzene liquid-crystalline polymers. J. Mater. Chem. 20, 117-120 (2010).

69 Kondo, M., Miyasato, R., Naka, Y., Mamiya, J., Kinoshita, M., Yu, Y., Barrett, C. J. \& Ikeda, T. Photomechanical properties of azobenzene liquid-crystalline elastomers. Liq. Cryst. 36, 1289 (2009).

70 Shimamura, A., Priimagi, A., Mamiya, J., Ikeda, T. YU, Y., Barrett, C.J. \& Shishido, A. Simultaneous analysis of optical and mechanical properties of cross-linked azobenzene-containing liquid-crystalline polymer films. ACS Appl. Mater. Interfaces 3, 4190-4196 (2011). 
71 Yamada, M., Kondo, M., Mamiya, J., Yu, Y., Kinoshita, M., Barrett, C. J. \& Ikeda, T. Photomobile polymer materials: towards light-driven plastic motors. Angew. Chem. Int Ed. 47, 4986-4988 (2008).

72 Yamada, M., Kondo, M., Miyasato, R., Naka, Y., Mamiya, J., Kinoshita, M., Shishido, A. Yu, Y., Barrett, C. J. \& Ikeda, T. Photomobile polymer materials-various threedimensional movements. J. Mater. Chem. 19, 60-62 (2009).

73 Cheng, F., Yin, R., Zhang, Y., Yen, C. \& Yu, Y. Fully plastic microrobots which manipulate objects using only visible light. Soft Matter 6, 3447-3449 (2010)
74 van Oosten, C. L., Bastiaansen, C. W. M. \& Broer, D. J. Printed artificial cilia from liquid-crystal network actuators modularly driven by light. Nat. Mater. 8, 677-682 (2009).

75 Naka, Y., Mamiya, J., Shishido, A., Washio, M. \& Ikeda, T. Direct fabrication of photomobile polymer materials with an adhesive-free bilayer structure by electronbeam irradiation. J. Mater. Chem. 21, 1681-1683 (2011).

76 Yoshino, T., Kondo, M., Mamiya, J., Kinoshita, M., Yu, Y. \& Ikeda, T. Three-dimensional photomobility of crosslinked azobenzene liquid-crystalline polymer films. Adv. Mater. 22, 1361-1363 (2010)

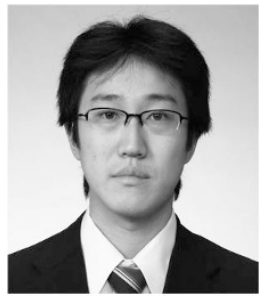

Jun-ichi Mamiya is currently an assistant professor of Chemical Resources Laboratory at Tokyo Institute of Technology. He received his BS degree under the guidance of the late Professor Yasuyuki Tanaka from Tokyo University of Agriculture and Technology in 1999 and his $\mathrm{PhD}$ degree under the supervision of Prof. Tomiki Ikeda at Tokyo Institute of Technology in 2004. After completing a postdoctoral researcher at RIKEN in 2005, he joined the staff of division of polymer chemistry in Tokyo Institute of Technology. He received the Award for Encouragement of Research in Polymer Science by the Society of Polymer Science, Japan (2011). His research interests are design of photofunctional polymer materials and application to light-driven polymer actuators. 